\title{
Dendritic Eph organizes dendrodendritic segregation in discrete olfactory map formation in Drosophila
}

\author{
Marie Anzo, ${ }^{1}$ Sayaka Sekine, ${ }^{1,4}$ Shirin Makihara, ${ }^{1,5,6}$ Kinhong Chao, ${ }^{1}$ Masayuki Miura, ${ }^{1,2}$ \\ and Takahiro Chihara ${ }^{1,3}$ \\ ${ }^{1}$ Department of Genetics, Graduate School of Pharmaceutical Sciences, The University of Tokyo, Bunkyo-ku, Tokyo 113-0033, \\ Japan; ${ }^{2}$ Japan Agency for Medical Research and Development (AMED)-CREST, Japan Agency for Medical Research \\ and Development, Chiyoda-ku, Tokyo 100-0004, Japan; ${ }^{3}$ Department of Biological Science, Graduate School of Science, \\ Hiroshima University, Higashi-Hiroshima, Hiroshima 739-8526, Japan
}

Proper function of the neural network results from the precise connections between axons and dendrites of presynaptic and postsynaptic neurons, respectively. In the Drosophila olfactory system, the dendrites of projection neurons (PNs) stereotypically target one of $\sim 50$ glomeruli in the antennal lobe (AL), the primary olfactory center in the brain, and form synapses with the axons of olfactory receptor neurons (ORNs). Here, we show that Eph and Ephrin, the well-known axon guidance molecules, instruct the dendrodendritic segregation during the discrete olfactory map formation. The Eph receptor tyrosine kinase is highly expressed and localized in the glomeruli related to reproductive behavior in the developing AL. In one of the pheromone-sensing glomeruli (DA1), the Eph cell-autonomously regulates its dendrites to reside in a single glomerulus by interacting with Ephrins expressed in adjacent PN dendrites. Our data demonstrate that the trans interaction between dendritic Eph and Ephrin is essential for the PN dendritic boundary formation in the DA1 olfactory circuit, potentially enabling strict segregation of odor detection between pheromones and the other odors.

[Keywords: Drosophila; Eph; Ephrin; dendrite; wiring]

Supplemental material is available for this article.

Received February 9, 2017; revised version accepted May 15, 2017.

The proper assembly of neural circuits during development is necessary for the formation of functional neural networks. One of the key steps for establishing a functional neural circuit is to construct a precise connection between the axons and dendrites of presynaptic and postsynaptic neurons, respectively. In the visual and auditory systems, neighboring neurons in the input field target the neighboring regions in the output field (Flanagan 2006; Kandler et al. 2009). In the olfactory systems of mammals and insects, the axons of the primary olfactory receptor neurons (ORNs) that express the same olfactory or ionotropic receptors converge to one specific glomerulus in the primary olfactory center (Couto et al. 2005; Sakano 2010; Silbering et al. 2011). The ORN axons form synaptic connections with dendrites of second-order neurons that also typically target one particular glomerulus among

Present addresses: ${ }^{4}$ Laboratory for Morphogenetic Signaling, RIKEN Center for Developmental Biology, Kobe, Hyogo 650-0047, Japan; ${ }^{5}$ Molecular Biology of Neural Development, Institut de Recherches Cliniques de Montréal, Montréal, Quebec H2W 1R7, Canada; ${ }^{6}$ Integrated Program in Neuroscience, McGill University, Montreal Neurological Institute, Montreal, Quebec H3A 2B4, Canada.

Corresponding author: tchihara@hiroshima-u.ac.jp

Article is online at http://www.genesdev.org/cgi/doi/10.1101/gad.297424. 117. those discretely distributed. Unlike in other sensory systems, there is less spatial correlation between axon and dendrite targeting in the olfactory system. Thus, the neuronal wiring in the olfactory system can be the most striking example of specific targeting achieved by both axons and dendrites among the neural targeting events during development. The previous studies have shown that the topographic mapping in the visual system and the neuronal wiring in the olfactory system are mostly governed by axon guidance (Flanagan 2006; Sakano 2010; Cang and Feldheim 2013). In comparison, dendrite targeting is far less understood not only due to the complex morphology and diversity of dendrites but also because its historical background has received little attention.

The Drosophila olfactory system is a suitable model to study the mechanisms underlying dendrite targeting (Fig. 1A; Jefferis and Hummel 2006; Hong and Luo 2014; Sakuma et al. 2014). The primary olfactory center, the

(C) 2017 Anzo et al. This article is distributed exclusively by Cold Spring Harbor Laboratory Press for the first six months after the full-issue publication date (see http://genesdev.cshlp.org/site/misc/terms.xhtml). After six months, it is available under a Creative Commons License (Attribution-NonCommercial 4.0 International), as described at http://creativecommons.org/licenses/by-nc/4.0/. 

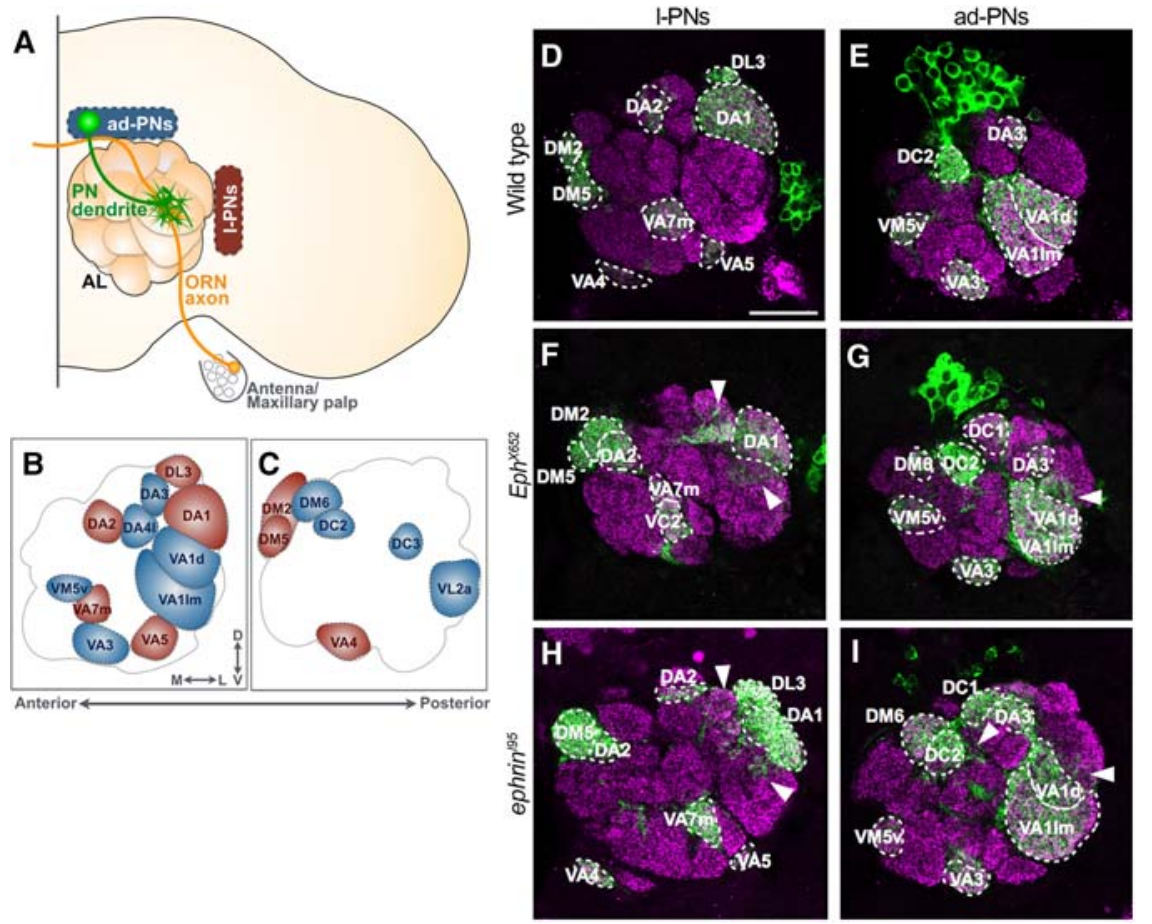

Figure 1. Loss of Eph or Ephrin exhibits ectopic dendritic projection. $(A)$ Schematic diagram of the Drosophila olfactory system. Axons of ORNs expressing the same types of olfactory receptor at the antenna or maxillary palp target one of $\sim 50$ glomeruli in the AL and specifically connect with corresponding dendrites of PNs. PNs are classified into four lineages according to the neuroblasts from which they are produced: anterodorsal PNs (adPNs; blue), lateral PNs (1-PNs; red), ventral PNs, and lateroventral PNs. $(B, C)$ Names of the 18 glomeruli (out of $\sim 50$ ) located at the anterior half of the AL. Each glomerulus is identifiable by its size, shape, and relative position. The glomeruli where dendrites of 1-PNs and ad-PNs innervate are colored in red and blue, respectively. $(D-I)$ Green indicates PN neuroblast clones labeled by $m C D 8$ GFP driven by GH146-Gal4-based MARCM. Magenta represents the presynaptic marker Bruchpilot. $(D, E)$ Wild-type neuroblast clones: 1-PNs $(D ; n=9)$ and ad-PNs $(E ; n=7) . \quad(F, G)$ 1-PNs $(F ; n=6)$ and ad-PNs $(G ; n=10)$ of $E p h^{X 652}$. Ectopic dendrite projections were observed (arrowheads). $(H, I)$ 1-PNs $(H ; n=4)$ and ad-PNs $(I ; n=7)$ of ephrin ${ }^{I 95}$. Ectopic dendrite projections were also observed (arrowheads). Bar, $25 \mu \mathrm{m}$.

antennal lobe (AL), consists of $\sim 50$ discrete structures called glomeruli that are identifiable from their shape, relative size, and position (Fig. 1B,C). Most of the projection neuron (PN) dendrites invade one particular glomerular space and form synapses with axons of a single ORN class. In addition, genetic tools such as mosaic analysis with a repressible cell marker (MARCM) allow us to label specific subsets of PNs at a single-cell resolution in vivo and simultaneously manipulate genes in the labeled neurons (Lee and Luo 1999).

By taking advantage of the Drosophila olfactory system, the cell surface molecules that regulate dendrite targeting have been gradually revealed. Cell surface molecules such as Semaphorin-1a (Sema-1a) and Toll-6 cell-autonomously regulate dendrite targeting along the dorsolateralventromedial axis and mediolateral axis, respectively (Komiyama et al. 2007; Ward et al. 2015). The PN dendrites determine their coarse position in the $\mathrm{AL}$ along the axes depending on the expression level of Sema-1a or Toll-6 therein. The leucine-rich repeat transmembrane protein Capricious (Caps) is differentially expressed in a subset of PNs and represents a mosaic pattern in the developing AL (Hong et al. 2009). The differential Caps expression cell-autonomously instructs glomerular-specific PN targeting, especially the segregation of Caps-positive and Caps-negative PN classes. These findings indicate that besides axial information, discrete determinants also provide positional information to the PN dendrites. Moreover, the cell adhesion molecule N-cadherin (Ncad) and immunoglobulin superfamily protein Dscam act as attractive or repulsive signals in most of the PN classes that restrict the dendritic field to the appropriate glomerular space (Zhu and Luo 2004; Zhu et al. 2006). In addition to these findings, we here found that the dendritic boundary formation between specific subtypes of PNs are instructed by cell surface molecules, Eph, and Ephrin.

The Eph receptor and its ligand, Ephrin, are the largest family of receptor tyrosine kinases (RTKs) and are widely conserved from invertebrates to mammals (Bossing and Brand 2002; Chin-Sang et al. 2002; Dearborn et al. 2002; Lisle et al. 2013; Kania and Klein 2016). Eph and Ephrin have been well studied as axon guidance molecules in retinotectal topographic mapping (Cang and Feldheim 2013). In the vertebrate tectum/superior colliculus (SC), the EphA/EphrinA and EphB/EphrinB countergradients are formed along the anterior-posterior and nasal-temporal axes, respectively. The axons of retinal ganglion cells (RGCs) determine their target field by recognizing the relative position based on the expression levels of their ligands at the tectum/SC. For example, the temporal RGC axon expressing EphA receives a repulsive signal from EphrinA expressed in the tectum/SC, which causes the temporal axon to avoid the posterior tectum/SC. In vertebrates, Ephrins are divided into two groups based on the type of membrane linkage: GPI-anchored EphrinAs (EphrinA1-6) and transmembrane EphrinBs (EphrinB1-3) (Lisle et al. 2013). Ephs are also divided into two subtypes depending on the affinity to Ephrins: EphAs (EphA1-8 and Eph10) bind to multiple EphrinAs, and, similarly, EphBs (EphB1-4 and Eph6) bind to multiple EphrinBs, with the exception of EphA4 binding to both EphrinAs and EphrinBs. Since both Ephs and Ephrins are membrane-bound 
proteins, the signal is essentially activated via contact-dependent cell-cell interaction. The bidirectional Eph/Ephrin signal works repulsively in a majority of the cases, although an adhesive response has also been described (Klein 2012; Kania and Klein 2016). Since Drosophila has only a single pair of Eph-Ephrin, we can exclude the overlapping function of their family members as is considered in vertebrate studies (Bossing and Brand 2002; Dearborn et al. 2002). Drosophila Eph shows 71\% identity with both vertebrate EphA3 and EphB2 (Scully et al. 1999), and Drosophila Ephrin has a vertebrate EphrinBlike cytoplasmic domain (Bossing and Brand 2002). A previous study of topographic mapping in the Drosophila visual system strongly suggested that Drosophila Eph/ Ephrin signal functions in an evolutionarily conserved fashion (Dearborn et al. 2002).

In this study, we found that Eph/Ephrin signal instructs dendrodendritic segregation during discrete olfactory map formation. Unlike Ncad or Dscam, which affects most of the PN classes, Eph/Ephrin signal selectively functions in only specific PN classes. High Eph RTK expression was observed specifically in the glomeruli associated with reproductive behavior in the developing AL. In addition, our genetic data indicate that Eph/Ephrin trans interaction between neighboring glomeruli plays a central role in local dendrodendritic segregation through bidirectional repulsive responses.

\section{Results}

Loss of Eph or Ephrin exhibits a dendritic spillover phenotype in PNs

We showed previously that Ephrin is involved in PN dendrite refinement through knockdown experiments using RNAi (Sekine et al. 2013). To examine how Eph and Ephrin function in dendrite targeting, we first observed morphologies of PN dendrites in an Eph- or ephrin-null mutant background by labeling PNs with GH146-Gal4-driven MARCM (Boyle et al. 2006). Many of the wild-type PNs are derived from either anterodorsal or lateral neuroblasts, and each PN dendrite converges to and resides in one specific glomerulus out of $\sim 50$ glomeruli according to $\mathrm{PN}$ classes (Fig. 1B-E). In $E p h^{X 652}$, most of the PN dendrites targeted the appropriate glomeruli, although some dendrites showed additional invasion to the adjacent glomeruli (Fig. 1F,G, arrowheads). Since only an ephrin hypomorphic mutant exists, we used the CRISPR/Cas9 system to generate a knockout line called ephrin ${ }^{I 95}$, which lacks the genomic region from the $5^{\prime}$ untranslated region (UTR) to the 3' UTR (Supplemental Fig. S1A). The dendritic spillover phenotype was also observed in ephrin ${ }^{I 95}$ (Fig. 1H,I, arrowheads), suggesting that both Eph and Ephrin are involved in the targeting processes of $\mathrm{PN}$ dendrites.

Eph is specifically expressed in the developing glomeruli associated with reproductive behavior

Next, we investigated the expression patterns of Eph and Ephrin during development. In Drosophila AL develop- ment, $\mathrm{PN}$ dendrites roughly target near the final targeting area before the arrival of ORN axons (Fig. 2A; Jefferis et al. 2004; Jefferis and Hummel 2006; Sakuma et al. 2014). Pioneering ORN axons first wrap around the AL and then start invading inside it ( $24 \mathrm{~h}$ after puparium formation [APF]) (Fig. 2B). The discrete synaptic structures called glomeruli are formed $\sim 50 \mathrm{~h} \mathrm{APF}$ and become matured during the subsequent pupal stage (Fig. 2C,D). A previous study showed ORN-derived Eph expression by Ephrin-Fc in situ labeling (Singh et al. 2013), which can detect only Eph receptors that have not bound to the endogenous ligand (Kao and Kania 2011). To monitor the amount and localization of the endogenous Eph protein, we generated a knock-in allele $(E p h-m y c)$ by inserting the myc sequence at the $3^{\prime}$ end of the Eph coding sequence using the CRISPR/Cas9 system (Supplemental Fig. S1B). The Ephmyc expression was confirmed by the Western blotting of pupal brain lysate and the immunohistochemistry of the pupal optic lobe at $50 \mathrm{~h}$ APF (Supplemental Fig. S1C-E" $E^{\prime \prime}$. The molecular weight of Eph-myc (knock-in allele) was comparable with that of Eph-HA overexpressed in S2 cells (Fig. 6C, below; Supplemental Fig. S1C). In addition, the expression pattern of Eph-myc in the pupal optic lobe was consistent with the previous report (Supplemental Fig. S1D,E; Dearborn et al. 2002). AntiMyc antibody staining revealed Eph expression inside the AL from the early stages of development $(\sim 18 \mathrm{~h}$ $\mathrm{APF})$, when the ORN axons have not yet invaded the AL (Fig. 2E, E',I,I'; Supplemental Fig. S2). As the development of local interneurons (LNs) depends on ORN axons (Chou et al. 2010), this weak but distinct signal is likely derived from $\mathrm{PN}$ dendrites. In addition, the pioneering ORN axons showed strong Eph expression (Fig. 2E, $\mathrm{E}^{\prime}, \mathrm{F}, \mathrm{F}^{\prime}, \mathrm{I}, \mathrm{I}^{\prime}, \mathrm{J}, \mathrm{J}^{\prime}$; Supplemental Fig. S2C-E"'). Of note, strong Eph-myc expression was observed in en route axon tract processes but not in the tip of the pioneering ORN axon (Supplemental Fig 2C-D"I. At $50 \mathrm{~h}$ APF, we found that the Eph expression was especially high in the DL3, DA1, VA1lm, and VL2a glomeruli, which are known to respond to the odors related to reproductive behavior (Fig. $2 \mathrm{G}, \mathrm{G}^{\prime}, \mathrm{K}, \mathrm{K}^{\prime}$; Supplemental Fig. S3A-D'; Ejima et al. 2007; Kurtovic et al. 2007; van der Goes van Naters and Carlson 2007; Grosjean et al. 2011; Liu et al. 2011; Lebreton et al. 2014; Lin et al. 2016). Interestingly, the expression was markedly low in the neighboring regions of the Eph-positive glomeruli (Supplemental Fig. S3A-D', arrowheads). We then asked whether PNs express Eph-myc. We knocked down Eph in PNs by using Eph-shRNA and GH146-Gal4 and found that PN-specific knockdown of Eph reduced Eph-myc signal in developing PNs, indicating that Eph is expressed in developing PNs (Supplemental Fig. S3E-G'). Together with the previous study showing ORN-derived Eph expression by Ephrin-Fc in situ labeling (Singh et al. 2013), we conclude that both PNs and ORNs express Eph at 50 $\mathrm{h}$ APF. The patterned expression observed at $50 \mathrm{~h}$ APF disappeared in the adult stage (Fig. $2 \mathrm{H}, \mathrm{H}^{\prime}, \mathrm{L}^{\prime} \mathrm{L}^{\prime}$ ).

On the other hand, the Ephrin expression was reported to be ubiquitous throughout the developing AL by antiEphrin antibody staining (Singh et al. 2013). We also tried to observe Ephrin expression by inserting the myc 

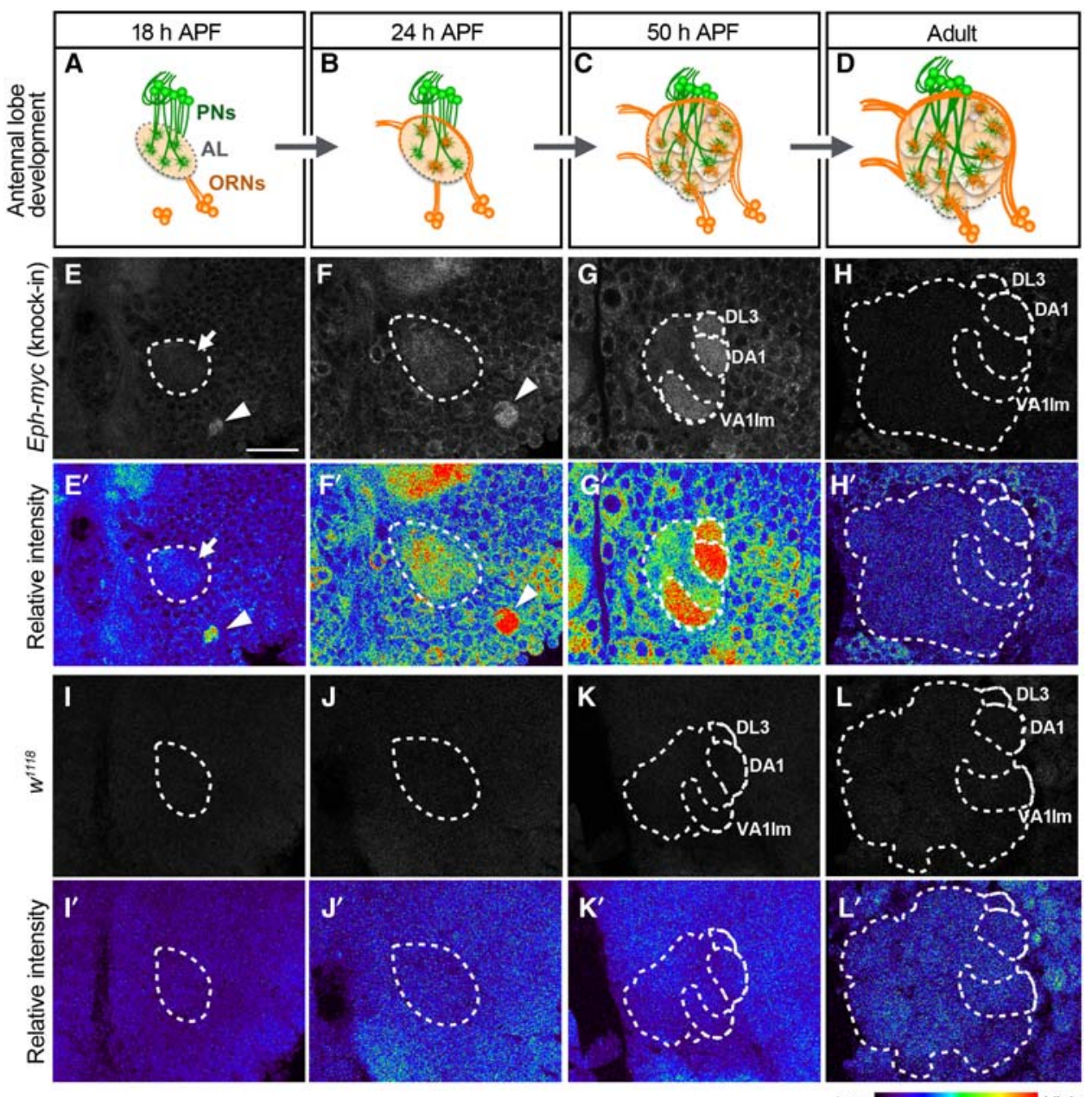

Low High

Figure 2. Eph is highly expressed in glomeruli related to reproductive behavior. $(A-D)$ Schematic views of the adult AL development during pupal stages. (A) PN dendrites roughly target near the final target area in the developing AL by $18 \mathrm{~h}$ APF. (B) Pioneering ORN axons reach and invade the AL. $(C, D)$ The synaptic structures called glomeruli are visible $\sim 50 \mathrm{~h} A P F(C)$ and undergo a maturation process by the adult stage $(D) .(E-H)$ Anti-myc staining was performed on $w_{; ;}$Eph-myc. $(E) n=7 .(F) n=10 .(G) n=7$. $(H) n=7$. $\left(E^{\prime}-H^{\prime}\right)$ Relative intensity of anti-myc staining shown in pseudocolors. Myc signal was observed inside the AL (the dotted circle was identified by costaining with anti-

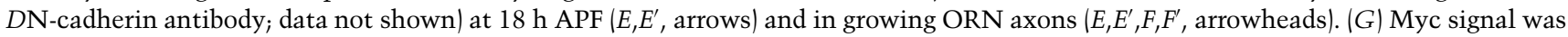
especially high at DL3, DA1, and VA1lm. $(H)$ The patterned expression disappeared in the adult stage. $(I-L)$ Anti-myc staining against $w^{1118}$ (negative control). $(I) n=6 .(J) n=9 .(K) n=8 .(L) n=10$. $\left(I^{\prime}-L^{\prime}\right)$ Relative intensity of anti-myc staining. Bar, $25 \mu \mathrm{m}$.

sequence at the $\mathrm{C}$ terminus of ephrin (ephrin-myc); however, it was barely detectable, probably because of its weak expression (data not shown). Based on these results and those from the mutant analyses indicating the cell-autonomous function of Eph in the PNs (described below), we conclude that Eph is expressed exclusively in the developing glomeruli associated with reproductive behavior.

Eph cell-autonomously instructs the DA1 lateral PN (1-PN) dendrites not to spill over to the neighboring glomeruli

As Eph showed preferential expression in a specific subset of glomeruli at $50 \mathrm{~h} \mathrm{APF}$, we next investigated how this expression functions in dendrite targeting. To further ex- amine the cell-autonomous role of Eph in specific classes of PNs during dendrite targeting, we used Eph-shRNA generated in our previous study (Sekine et al. 2013). When Eph was knocked down in the PNs derived from lateral neuroblasts (1-PNs), the dendrite spilled over from DA1 and DL3 glomeruli, where the Eph was highly expressed during development (Fig. 3A, arrowheads). We then carefully quantified the glomerular invasion of 1 PNs expressing Eph-shRNA and found that those dendrites ectopically invaded the DA3, DA4l, VA1d, DM6, DC3, and VL2ap glomeruli (Fig. 3B). The glomeruli receiving ectopic dendrite innervation with $>80 \%$ penetrance (DA4l, 81.8\%; VA1d, 100\%; and DC3, 100\%) were all adjacent to the DAl glomerulus (Figs. 1B,C, 3B). The dendrites of wild-type DA1 1-PNs converged to the DA1 without any spillover (Fig. 3E,F). In contrast, the DA1 


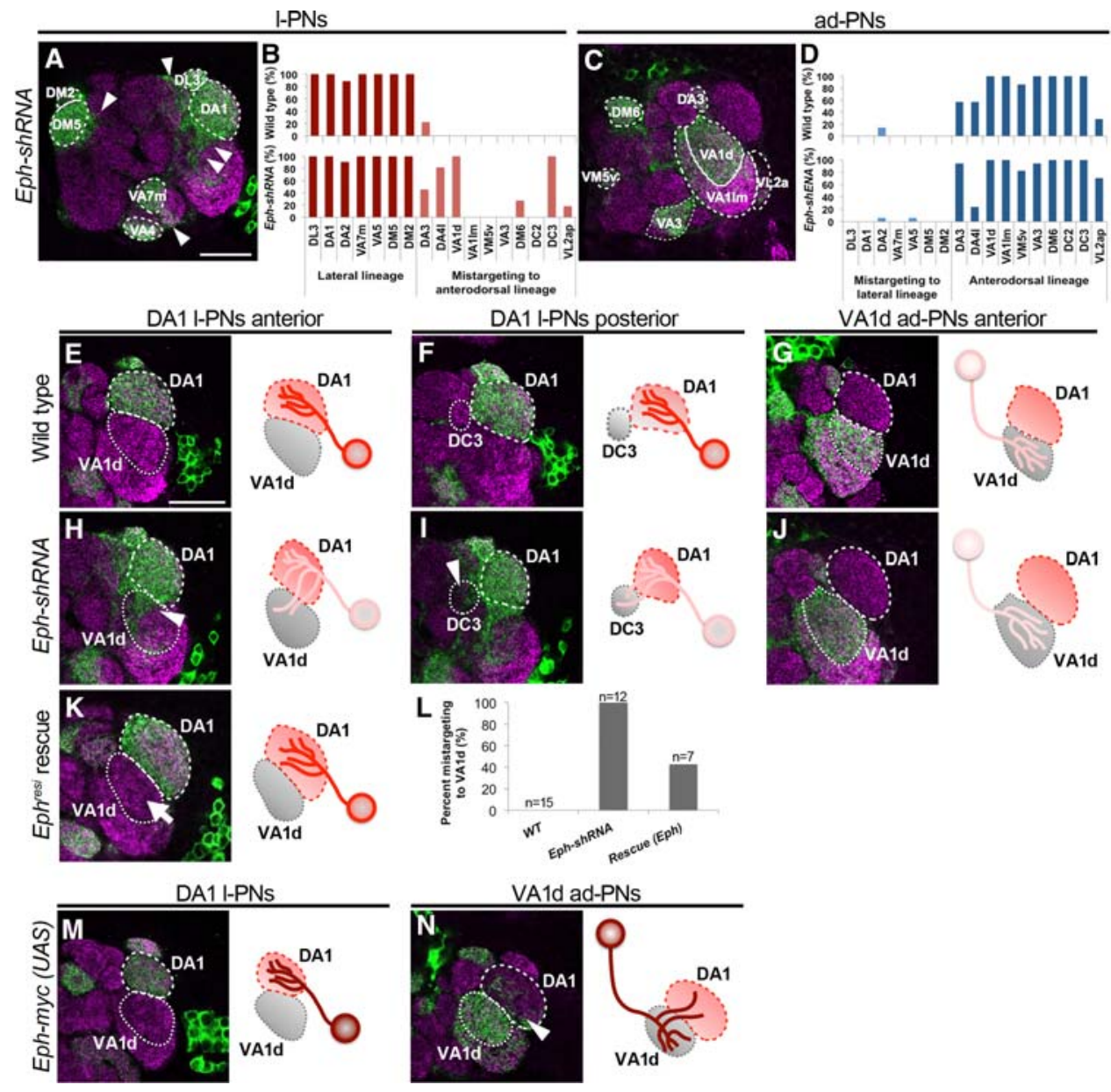

Figure 3. Eph expression is cell-autonomously required for DA1 PNs to not spill over to the adjacent glomeruli. (A) GH146-Gal4-driven MARCM clone of 1-PNs expressing Eph-shRNA and $m C D 8$-GFP. Ectopic dendrite projections are indicated by arrowheads. $(B)$ Quantification of glomerular innervation specificity of 1-PN dendrites of control (top row; $n=9$ ) or Eph-shRNA-expressing (bottom row; $n=11$ ) PNs. The $X$-axis represents glomeruli that l-PN dendrites innervated. Bars colored in lighter red indicate the glomeruli where ectopic dendritic invasions were observed. (C) GH146-Gal4-driven MARCM clone of ad-PNs expressing Eph-shRNA and $m C D 8$-GFP. (D) Quantification of ad-PN glomerular innervation of control (top row; $n=7$ ) or Eph-shRNA-expressing (bottom row; $n=17$ ) PNs. The $X$-axis represents glomeruli that ad-PN dendrites innervated. Bars in lighter blue indicate the ad-PN dendrites mistargeting to the glomeruli that are supposed to be the targets of lateral lineage PNs. $(E-G)$ Magnified view of a single section of the glomerular boundary between DA1 and neighboring glomeruli in the wild type. l-PNs (anterior section in $E$, posterior section in $F$ ) and ad-PNs (anterior section in $G$ ) are labeled by the clonal expression of $m C D 8$-GFP (green). (H-J) Magnified view of the glomerular boundary between DAl and neighboring glomeruli with PNs expressing Eph-shRNA. (H,I) DA1 dendritic spillover was observed from DA1 to VA1d and DC3 glomeruli (arrowheads). (J) Eph knockdown in VA1d ad-PNs did not cause VA1d dendritic spillover to DA1. (K) 3xFlag-Eph ${ }^{\text {resistant }}$-HA expression rescued the dendritic spillover phenotype observed in DA1 1-PNs expressing Eph-shRNA (arrow). $n=7$. (L) Quantification of $E, H$, and $K$. ( $M$ ) Overexpression of Eph-myc in DA1 l-PNs did not cause DA1 dendritic spillover. $n=7$. $(N)$ In contrast, overexpression of Eph-myc in VAld adPNs caused VA1d dendritic spillover to DA1 glomeruli (arrowhead). $n=8$. Magenta represents the presynaptic marker Bruchpilot. Bar, 25 $\mu \mathrm{m}$.

1-PN dendrites expressing Eph-shRNA spilled over to its adjacent glomeruli, such as VAld and the more posterior DC3 glomeruli (Fig. 3H,I). Since the VA1d glomerulus is located most anteriorly and its glomerular boundary is easily distinguishable from the others, we focused here on the boundary between the DAl and VAld glomeruli. This spillover phenotype was observed from $50 \mathrm{~h} \mathrm{APF}$, suggesting that Eph functions at the dendritic segregation process (Supplemental Fig. S4). The MARCM expression of Flag-Eph ${ }^{\text {resistant }}$-HA (a Flag- and HA-tagged form of Eph resistant to Eph-shRNA) rescued the DAl dendritic spillover phenotype of the l-PNs expressing Eph-shRNA (Fig. 3K,L). Thus, Eph expression is cell-autonomously required for the DA1 1-PN dendrites not to spill into its adjacent glomeruli.

In contrast, Eph knockdown in the PNs, including the VA1d and DC3 PNs, did not cause any spillover phenotype to DA1 glomerulus (Fig. 3C,D,G,J). This was inconsistent with the observations in the $E p h^{X 652}$ mutant (Fig. 1G arrowhead), likely owing to the difference between the null mutant $\left(E p h^{X 652}\right)$ and shRNA-mediated knockdown mutant. Alternatively, it may reflect the 
non-cell-autonomous function of Eph in the DA1 1-PNs to the adjacent dendrites such as the VAld anterodorsal PN (ad-PN) dendrites (see the Discussion for more details). Altogether, the sensitivity to Eph-shRNA positively correlated with endogenous Eph expression level, suggesting the importance of Eph signals especially in the PNs that highly express Eph.

\section{Differential Eph expression levels between DA1 and VA1d PNs enable dendritic segregation}

We showed that high Eph expression is required in the DA1 1-PN to keep their dendrites in the DA1 glomerulus and that the Eph expression is low in the neighboring glomeruli such as the VA1d. Therefore, we hypothesized that the difference in Eph expression levels between the DA1 (high) and VAld (low) could suppress the dendritic spillover to neighboring glomeruli. To examine this idea, we manipulated the Eph expression levels between DA1 and VAld by overexpressing Eph. Eph overexpression in the DA1 1-PNs, which originally showed high Eph expression, did not result in any spillover $(n=7)$ (Fig. 3M). In contrast, Eph overexpression in the VAld ad-PNs caused VAld dendritic spillover into the DA1 glomerulus with $100 \%$ penetrance $(n=8)$ (Fig. $3 \mathrm{~N})$. To further test whether VA1d ad-PN concentration of Eph controls dendritic segregation, we altered the Eph-myc (UAS) expression level by changing the temperature of the culturing condition to either $25^{\circ} \mathrm{C}$ or $18^{\circ} \mathrm{C}$ (Supplemental Fig. S5). Based on the features of the Gal4/UAS system, we could lower the expression level of the transgene under the UAS sequence by lowering the temperature of the culturing condition. The spillover phenotype of the Eph-overexpressing ad-Nb MARCM clone was ranked blindly according to the spillover level of VA1d ad-PN dendrites to the DA1 glo- merulus. The VAld dendritic spillover phenotypes of $25^{\circ} \mathrm{C}$ flies were significantly more severe than those of $18^{\circ} \mathrm{C}$ flies, suggesting that the severity of the spillover phenotype correlates with the expression level of misexpressed Eph in VAld ad-PNs. These data indicate that Eph expression is required to be kept low in the VAld ad-PN dendrites that reside in the VAld glomerulus. Moreover, the spillover from Eph-overexpressing VAld preferentially invaded the DA1 glomerulus but not the other adjacent glomeruli whose Eph expression levels are low. These data suggest that VAld dendrites discriminate themselves and DA1 dendrites by detecting the differences in Eph levels on the boundary between VA1d and DA1 glomeruli.

\section{Ephrin in VA1d is non-cell-autonomously required for the DA1 dendrites to not spill into VA1d dendrites}

We presented that the high Eph expression in the DA1 1PNs is necessary for the formation of a proper DA1VA1d dendritic boundary. Next, we investigated what the DA1 dendritic Eph recognizes as a ligand in order to keep away from its adjacent glomeruli. We focused on Ephrin as an interacting partner for the DA1 dendritic Eph. We hypothesized that the interaction between the DA1 dendritic Eph and its adjacent dendritic Ephrin works repulsively, thus preventing them from getting entangled. We analyzed wild-type DA1 1-PN behavior when ephrin was knocked down in the VAld ad-PNs using independent double MARCM (Fig. 4A; Potter et al. 2010). As illustrated in Supplemental Figure S6, in addition to ephrin knockdown in the ad-PNs using GH146-Gal4-driven MARCM, we also used GH146-QF-driven MARCM to label wild-type 1-PNs in the same individual. The ALs incidentally bearing a combination of ephrin-shRNA-

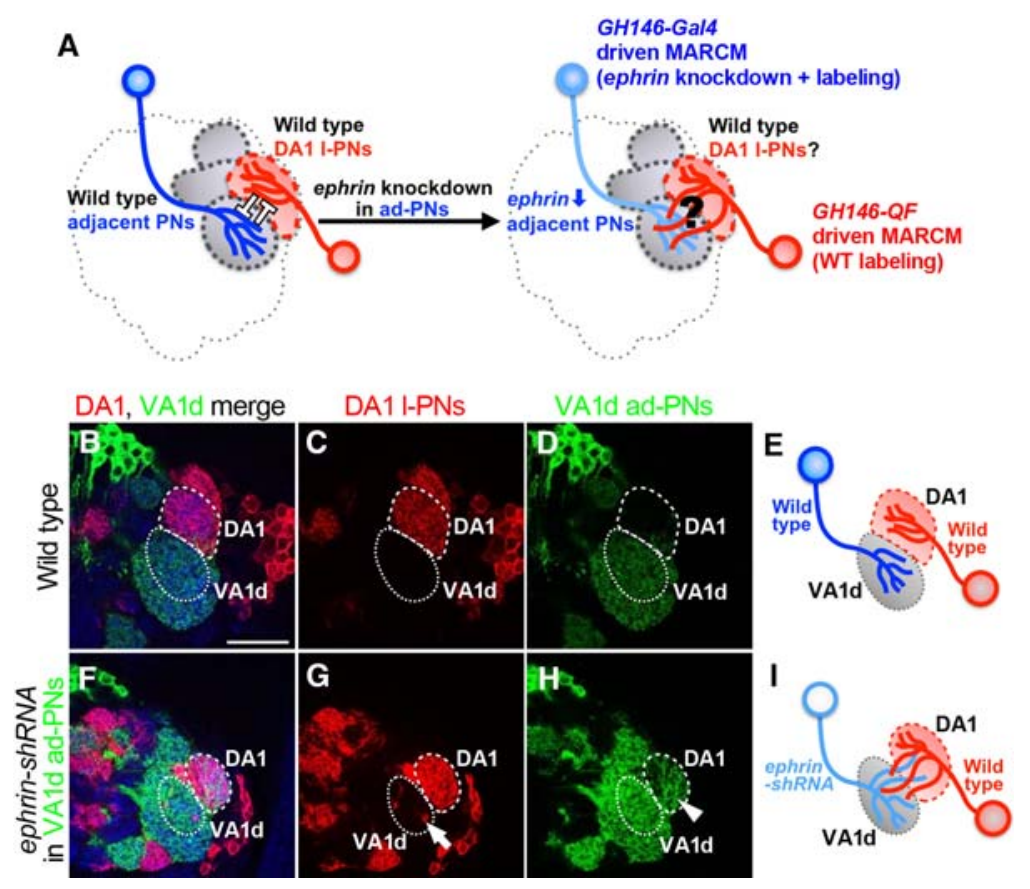

Figure 4. DA1-PN dendritic Eph recognizes adjacent PN Ephrin as a ligand. (A) Drawings showing the hypothesis that DA1 PN dendrites recognize Ephrin on the dendrites of adjacent glomeruli to stay in DA1. The behavior of wild-type DA1 PN dendrites was analyzed when ephrin was knocked down in surrounding ad-PNs such as VAld PNs. $(B-E)$ Wild-type DA1 1-PNs and VAld ad-PNs were labeled by mtdTomato-HA and $m C D 8-G F P$, respectively. $n=7$. The proper dendritic boundary was formed between DA1 1-PNs stained with anti-HA antibody $(C)$ and VA1d ad-PNs stained with anti-GFP antibody $(D) .(F-I)$ Wild-type DA1 1-PNs and ephrin-shRNA-expressing VAld ad-PNs were labeled simultaneously. $n=7$. $(G)$ Wild-type DA1 1-PNs spilled over to the adjacent glomeruli such as the VA1d glomerulus in $100 \%$ penetrance (arrow; seven out of seven). (H) ephrin-shRNA expression in VA1d ad-PNs cell-autonomously caused dendritic spillover into DA1 (arrowhead). Blue represents $D \mathrm{~N}$-cadherin staining. Bar, $25 \mu \mathrm{m}$. 
expressing ad-PNs (with mCD8-GFP) and wild-type 1-PNs (with mtdTomato-HA) as a result of $h s-F L P$-promoted mitotic recombination were analyzed. Of the $>6000$ brains dissected, we collected seven individuals for each of the experimental and control groups (Fig. 4B-I). In the control group (wild type), the DA1-VA1d PN boundary was properly formed (Fig. 4B-E). Specifically, the DA1 1-PNs never spilled into the adjacent glomeruli (Fig. 4C). When ephrin was knocked down in the ad-PNs, the wild-type DAl 1-PNs spilled into the adjacent glomeruli, including the VA1d (Fig. 4G, arrow), suggesting that ephrin knockdown in ad-PNs non-cell-autonomously affected the dendrites of the wild-type DA1 1-PN. Interestingly, ephrin knockdown in the ad-PNs also cell-autonomously affected the dendrites of the VAld ad-PNs; the VAld PN dendrites spilled into the DA1 glomerulus (Fig. $4 \mathrm{H}$, arrowhead). These data strongly suggest that dendritic Eph in the DA1 1-PNs recognizes the dendritic Ephrin in the adjacent ad-PNs as a ligand in order to prevent spillover to the adjacent glomeruli.

\section{Ephrin is cell-autonomously required for the VA1d dendrites to not spill into the DA1 glomerulus}

To further investigate the cell-autonomous role of Ephrin in $\mathrm{PN}$ dendrite targeting, we analyzed the projection patterns of the PNs under the ephrin knockdown condition in more detail. The knockdown of ephrin in the ad-PNs caused VAld ad-PN dendritic spillover into the DA1 glomerulus (Fig. 5A), as shown in the earlier independent double-MARCM experiment. However, ephrin knockdown in the DA1 1-PNs did not show any spillover phenotype (Fig. 5B), suggesting that Ephrin is also required in a class-specific manner in spite of the ubiquitous expression

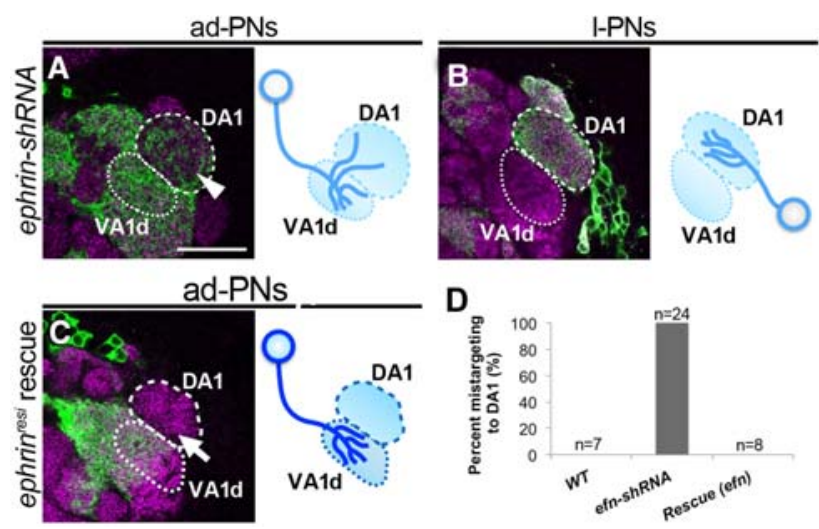

Figure 5. Ephrin expression is cell-autonomously required in VAld PN dendrites to prevent spillover to DA1. $(A, B)$ PNs expressing ephrin-shRNA and mCD8-GFP driven by GH146-Gal4based MARCM. (A) VAld ad-PNs spilled over into the DAl glomerulus as in Figure 3I (arrowhead). $n=24$. (B) ephrin knockdown in DA1 1-PNs did not cause dendritic spillover to VA1d. $n=6 .(C)$ ephrin $^{\text {resistant }}$-myc expression cell-autonomously rescued the dendritic spillover phenotype observed in PNs expressing ephrin-shRNA (arrow). $n=8$. Magenta represents the presynaptic marker Bruchpilot. $(D)$ Quantification of $A-C$. Bar, $25 \mu \mathrm{m}$. in the AL (Singh et al. 2013). Although the effect of ephrinshRNA lines is confirmed (Figs. 4F-I, 5A), we cannot exclude the possibility that the ephrin-shRNA lines are not able to adequately knock down ephrin expression in DA1 1-PNs. The VA1d ad-PN dendritic spillover phenotype was fully rescued by expressing ephrin ${ }^{\text {resistant }}$-myc (Fig. 5C,D), indicating that Ephrin functions cell-autonomously in the VAld dendrites to avoid getting entangled with the DA1 dendrites.

\section{Trans interaction between DA1 dendritic Eph and VA1d dendritic Ephrin is required for dendritic boundary formation}

Our data suggested that Eph and Ephrin cell-autonomously regulate the DA1 1-PNs and VA1d ad-PNs, respectively, in order to keep their dendrites in their own glomeruli and not spill over into the adjacent glomeruli. This implies that Eph and Ephrin interact in trans between the dendrites of the DA1 1-PN and VAld ad-PN, respectively. To further investigate the requirement of trans interaction of the DA1 dendritic Eph and VA1d dendritic Ephrin for dendritic boundary formation, we performed the following experiments. The predicted Drosophila Eph protein bears evolutionarily conserved domains, including the ligand-binding domain (LBD), cysteine-rich domain, fibronectin type III domains, transmembrane domain, tyrosine kinase domain, and a sterile a motif (SAM) (Fig. 6A; Dearborn et al. 2002). We generated a UAS construct with a truncated form of Eph lacking the LBD $(\triangle \mathrm{LBD})$, $E p h^{\triangle L B D}-H A$ (Carvalho et al. 2006). Next, we performed in vitro experiments to test whether this deletion in the Eph sequence abolishes the ability to bind to Ephrin in trans. We cotransfected S2 cells with either UAS-Eph$H A$ (wild-type Eph) or UAS-Eph ${ }^{\triangle L D D}-H A$ along with actin-Gal4. We immunostained the cells and tested their binding affinity to the Ephrin-Fc probe. The Ephrin-Fc probe bound strongly to wild-type Eph-HA and barely bound to $\mathrm{Eph}^{\triangle \mathrm{LBD}}$-HA (Fig. 6B). We further tested the trans Eph-Ephrin interaction using a coimmunoprecipitation experiment. Both the expression of Eph-HA and the expression of $\mathrm{Eph}^{\triangle \mathrm{LBD}}$-HA were detected in lysate, although $\mathrm{Eph}^{\triangle \mathrm{LBD}}$-HA did not bind to Ephrin-Fc (Fig. 6C). Both the immunostaining and coimmunoprecipitation experiments indicate that $\mathrm{Eph}^{\triangle \mathrm{LBD}}$-HA lacks the ability to bind to Ephrin in trans. To examine whether the trans interaction between the DA1 1-PNs and VA1d ad-PNs is necessary for the boundary formation, we performed a MARCM rescue experiment by using $E$ ph ${ }^{\text {resistant, }} \triangle \mathrm{LBD}_{-}$-HA. As we expected, the expression of Eph ${ }^{\text {resistant, } \triangle L B D}-H A$ could not rescue the DA1 1-PN dendritic spillover phenotype caused by Eph knockdown in the 1-PNs (Fig. 6D). In vertebrates, the E129K mutation in EphrinA5 rendered it incapable of binding to EphA3 in trans (Carvalho et al. 2006). We generated Drosophila Ephrin ${ }^{\mathrm{E} 320 \mathrm{~K}}$ that corresponded well with the vertebrate EphrinA $5^{\mathrm{E} 129 \mathrm{~K}}$ and performed a MARCM rescue experiment using Ephrin ${ }^{\mathrm{E} 320 \mathrm{~K}}$ to re-examine the requirement of the trans interaction in vivo. The expression of ephrin ${ }^{\text {resistant, } E 320 K}$-myc also could not rescue the VAld ad-PN dendritic spillover phenotype 
A
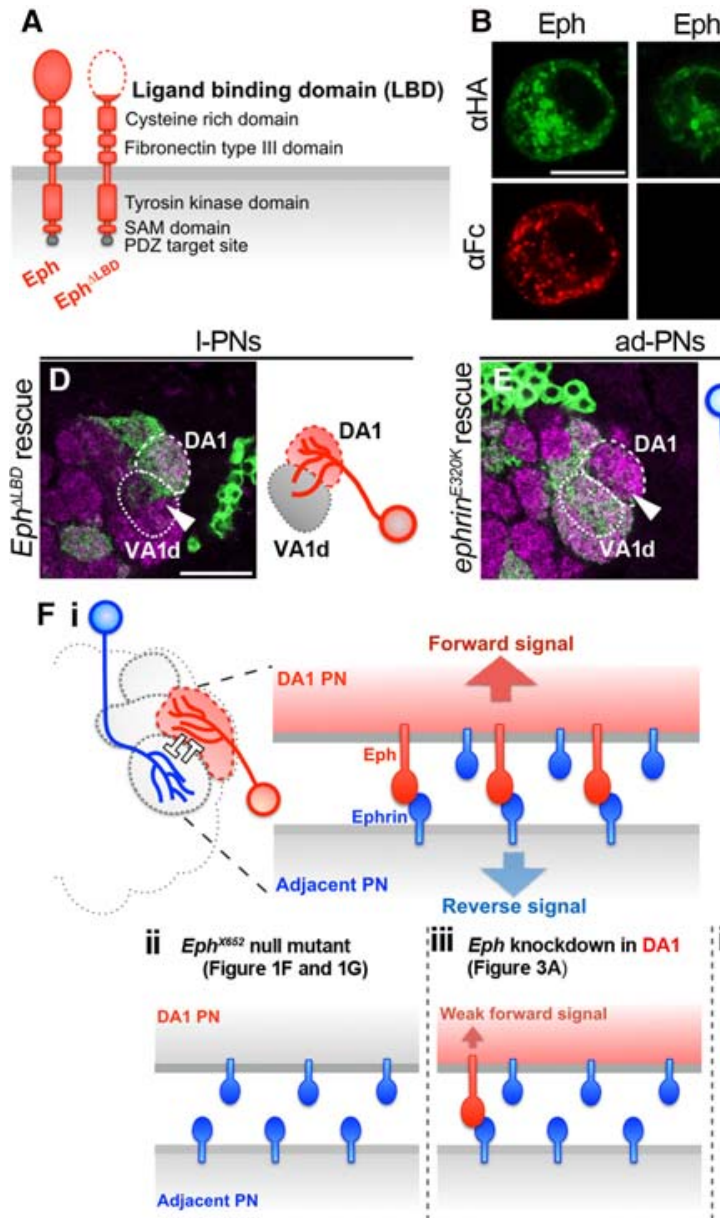

iii Eph knockdown in DA (Figure 3A)

\begin{tabular}{ll|c|c}
\hline $\begin{array}{l}\text { Phenotype } \\
\text { DA1 dendrite }\end{array}$ & Spillover & Spillover & n. d. \\
\hline VA1d dendrite & Spillover & n. d. & No phenotype
\end{tabular}
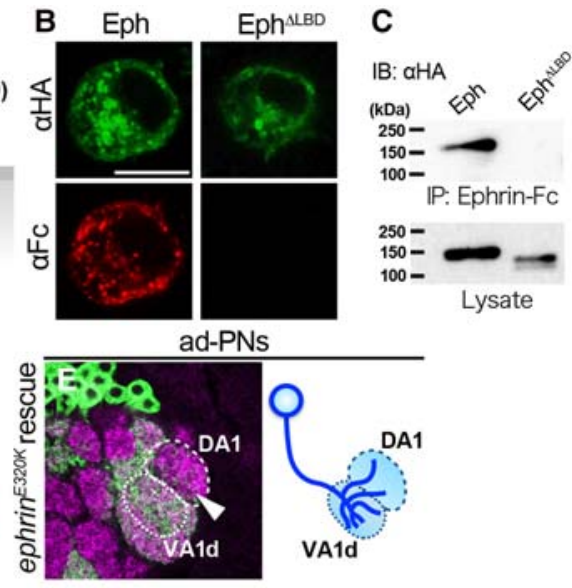

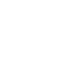

$$
\text { sy }
$$
marker Bruchpilot. Bar, 25 um. $(E)$ $-H A$ overexpression could not

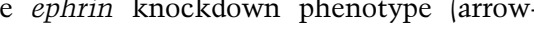
head). $n=13,100 \%$ penetrance. ( $F$, panel i) A model for dendrodendritic segregation between DA1 and adjacent glomeruli such as VAld by Eph/Ephrin signaling. As the result of trans interaction between DA1 dendritic Eph and adjacent dendritic Ephrin, bidirectional repulsive Eph/Ephrin signaling may run in dendrites of each side; thus, they segregate each other and form a proper dendritic glomerular boundary. (Panels ii-iv) Summary of the expected Eph/Ephrin signaling (schemes above) and observed phenotypes (below) in the $E p h^{X 652}$-null mutant (panel ii), Eph knockdown in DAl PN dendrites (panel iii), and Eph knockdown in VAld PN dendrites (panel iv). See the Discussion for more details. caused by ephrin knockdown in the ad-PNs (Fig. 6E). Thus, we conclude that the trans interaction between the DA1 dendritic Eph and VA1d dendritic Ephrin is important for the dendritic boundary formation between the DA1 and an adjacent glomerulus, such as VAld.

\section{Discussion}

How the robust specificity of the neural wiring is achieved is a fundamental question that must be answered in order to understand how functional neural networks are formed. The olfactory system is a striking example of wiring specificity, as the neurons within have to form synapses with their appropriate partners at anatomically discrete olfactory information channels called the glomeruli. In the initial step of the olfactory map formation in Drosophila, the coarse targeting of PN dendrites is instructed by the graded expression of Sema-1a (Komiyama et al. 2007). Next, the discrete determinants Caps and Tartan instruct the class-dependent glomerular targeting of PN dendrites based on their mosaic expression (Hong et al. 2009). The molecules expressed in mosaic patterns con- fine relatively broad subsets of neurons to innervate their cognate field. Our study provides another striking example of the mechanism underlying neuronal wiring specificity in that Eph is expressed in a very limited subset of neurons and acts locally to segregate discrete glomerular circuits through trans interactions with Ephrin on the dendrites of adjacent glomeruli.

\section{Eph/Ephrin segregation model}

Our study demonstrates that the trans interaction between the DA1 dendritic Eph and Ephrin on the adjacent dendrites is required for proper dendritic boundary formation. How can this be possible considering the patterned expression of Eph and the ubiquitous expression of Ephrin in the developing AL? We propose that the restricted expression of Eph in the DA1 dendrites could effect the activation of differential signal transduction between the dendrites in the DA1 and the adjacent glomeruli even though Ephrin is expressed ubiquitously throughout the developing AL. As the result of the trans interaction between the DA1 dendritic Eph and the adjacent dendritic 
Ephrin, the Eph forward signal seems to be transmitted to the Eph-expressing DA1 1-PNs, and the Ephrin reverse signal seems to be transmitted to the adjacent PNs (Fig. 6F, panel i). This trans interaction model involving Eph forward and Ephrin reverse signals also fits well with our result that the Eph-null mutant $\left(E p h^{X 652}\right)$, but not EphshRNA expression in the VAld ad-PNs, exhibited dendritic spillover from the VA1d to the DA1 glomerulus (Figs. 1G, 3J). The loss of Eph in the Eph ${ }^{X 652}$ mutant could weaken the Ephrin reverse signal in addition to the Eph forward signal, resulting in dendritic spillover from both the DA1 and VA1d dendrites (Fig. 6F, panel ii). In contrast, the cell-autonomous reduction of Eph in the VAld dendrites with Eph-shRNA has no way to reduce the Ephrin reverse signal (Fig. 6F, panel iv). In vertebrates, the trans Eph-Ephrin interaction leads to the formation of higher signaling clusters (Seiradake et al. 2010; Kania and Klein 2016). Oligomers of Ephs and Ephrins are terminated by bidirectional transendocytosis or its cleavage by ADAMtype proteases, leading to the activation of Rho family GTPases. This activation of Rho family GTPases by Eph/Ephrin oligomerization modulates actin cytoskeletal dynamics, which induce cell-cell repulsion in the most cases (Klein 2012). Hence, we propose the segregation model as follows. The trans interaction between the DA1 dendritic Eph and the adjacent dendritic Ephrin results in a bidirectional repulsive Eph forward and Ephrin reverse signal running in both DA1 and VA1d PNs, respectively; thus, they segregate from each other, thereby forming a proper dendritic glomerular boundary. Additional studies may be required to describe the molecular features of the Drosophila Ephrin reverse signal in the future.

\section{Glomerular boundary formation by Eph/Ephrin} for the pheromone-sensing circuit

When we knocked down Eph, the dendritic spillover phenotype was observed specifically near the DA1 and DL3 glomeruli. Why is the Eph/Ephrin system used to form the dendritic boundary of such specific glomeruli? Among 50 glomeruli, the DA1, DL3, VA1lm, and VL2a glomeruli exhibited high Eph expression during development, holding the line against the other glomeruli by their unique function. Unlike other glomeruli, the DA1, DL3, VA1lm, and VL2a glomeruli receive inputs from the axons of ORNs (Or67d, Or65a, Or47b, and Ir84a, respectively) dedicated to sensing odors related to reproductive behavior, such as pheromones and food-derived odors promoting male courtship behaviors (Ejima et al. 2007; Kurtovic et al. 2007; van der Goes van Naters and Carlson 2007; Grosjean et al. 2011; Liu et al. 2011; Lebreton et al. 2014; Lin et al. 2016). Among them, Or67d, the primary neuron of the DA1 olfactory circuit, detects Drosophila male-specific pheromone 11 cis-vaccenyl acetate (cVA) and triggers sex-specific courtship behavior in both male and female flies (Kurtovic et al. 2007). In addition, the DA1 PNs show sexually dimorphic neural circuitry (Datta et al. 2008). The primary neuron of DL3 (Or65a) also responds to cVA when flies are exposed to it for a long period. DL3 olfactory neurons suppress pheromonal activa- tion of DA1 olfactory neurons (Liu et al. 2011; Lebreton et al. 2014). Furthermore, as in Drosophila, the pheromone-sensing organs of Manduca sexta (macroglomerular complex [MGC]) and mice (vomeronasal organ [VNO]) express Ephrin, and their adjacent regions express Eph (Knoll et al. 2001; Kaneko and Nighorn 2003). Although Drosophila shows an opposite pattern of Eph/Ephrin (Eph in the pheromone-sensing circuit and Ephrin in the adjacent region), the same signaling machinery seems to have a conserved role in glomerular boundary formation across species. Interestingly, the mouse accessory olfactory bulb receiving input from the VNO, the moth MGC, and the Drosophila pheromone sensory glomeruli are all clustered and located dorsally to the other ordinary glomeruli in the mouse main olfactory bulb and the moth/ Drosophila ALs, respectively (Kaneko and Nighorn 2003; Fishilevich and Vosshall 2005; Sakurai et al. 2014). This conserved anatomical feature also suggests a notion that a unique signaling pathway is playing a role to secure the strict segregation between the pheromone-sensing circuits and the other olfactory circuits. Taken together, we hypothesize that the reproductive behavior circuit is highly specific and segregated from the others using the Eph/ Ephrin signal. Since Eph and Ephrin are both membranebound proteins, the signal is activated in a contact-dependent manner. In addition, the bidirectional signal transduction characteristic of the Eph/Ephrin signal system is reasonable for the local dendrodendritic segregation. It is possible that proper segregation in a dendrite level is necessary for building a well-organized neural network, thus allowing the optimal transfer of pheromone-related information to a higher brain center while controlling the courtship behavior.

\section{Materials and methods}

\section{Fly strains}

Flies were maintained under standard laboratory conditions $\left(25^{\circ} \mathrm{C}\right)$. The following mutants and transgenic lines were used: $E_{p h}{ }^{X 652}$ (Boyle et al. 2006), ephrin ${ }^{I 95}$, Eph-myc (knock-in), UASEph-shRNA (Sekine et al. 2013), UAS-Eph resistant-myc, UAS-Eph-myc, UAS-ephrin-shRNA (Sekine et al. 2013), UASephrin resistant-myc (Sekine et al. 2013), UAS-Flag-Eph ${ }^{\text {resistant }}{ }_{\text {- }}$ $H A$, UAS-Eph ${ }^{\triangle L B D}-H A$, UAS-Eph ${ }^{\text {resistant, } \triangle L B D}-H A$, and UAS-ephrin resistant, E320K-myc (generated in this study). The genotypes of Drosophila melanogaster used in each experiment are listed in Supplemental Table S1.

\section{Generation of knockout and knock-in constructs by CRISPR/Cas9}

The ephrin ${ }^{195}$ and Eph-myc alleles were generated in this study according to the methods available on flyCRISPR (http:// flycrispr.molbio.wisc.edu/protocols). As shown in Supplemental Figure S1, the sequence between the 5' UTR and the 3' UTR of the ephrin gene was knocked out to generate ephrin ${ }^{I 95}$ (Supplemental Fig. S1A), and the c-Myc tag was inserted at the $3^{\prime}$ end of the Eph coding sequence to generate Eph-myc (Supplemental Fig. S1B) through CRISPR/Cas9-mediated homology-directed repair. We injected two guide RNA vectors and a donor vector into vasa-Cas9 flies (Bloomington Drosophila Stock Center, no. 51324). The DNA fragments for the guide RNAs were subcloned 
in the BbsI-digested U6b-sgRNA-short (a kind gift from N. Perrimon) (Ren et al. 2013) vector. The following primers were annealed to generate the DNA fragments for guide RNAs: ephrin ${ }^{195}$-1 (5'-CTTCGATGTACCAAAAAAGGAAGA-3' and 5'-AAACTCTTCCTTTTTTGGTACATC-3'), ephrin ${ }^{195}$-2 (5' -CTT CGGAATCAAATGATATTAATT- $3^{\prime}$ and $5^{\prime}$-AAACAATTAATA TCATTTGATTCC-3'), Eph-myc-1 (5'-CTTCGACGGTAATCA TATTTTGGA-3' and 5'-AAACTCCAAAATATGATTACCG TC-3'), and Eph-myc-2 (5'CTTCGTACGTAAGGTGCGG TATTC-3' and 5'-AAACGAATACCGCACCTTACGTAC-3'). The $5^{\prime}$ and $3^{\prime}$ homology arms were inserted into the NotI and SpeI sites of the $p H D$-DsRed-attP (a kind gift from S. Kondo) (Gratz et al. 2014), respectively, by In-Fusion (Clonetech). For the Eph-myc, the 7xMyc sequences were also inserted in addition to the $5^{\prime}$ homology arm by three-fragment In-Fusion. The homology arms were amplified by PCR using the following primers: ephrin ${ }^{I 95} 5^{\prime}$ homology arm (forward primer, 5'-TGCATGCT AGCGGCCGCAATCGGAATTCTATCCAA-3', and reverse primer, 5'-TGCATGCTAGCGGCCGCAATCGGAATTCTAT CCAA-3'), ephrin ${ }^{I 95} 3^{\prime}$ homology arm (forward primer, 5'-ATA GAAGAGCACTAGGAGAGGTTGGAATCAAATGA-3', and reverse primer, 5'-GGAGATCTTTACTAGGGTAGCACAAG ATGCCTC-3'), Eph-myc 5' homology arm (forward primer, 5'-TGCATGCTAGCGGCCGCATACACAACTCGGGTAA-3', and reverse primer, $5^{\prime}$-ATACCGTCGACCTCGTATTATAGTG TCGAGTTGCC-3'), and Eph-myc 3' homology arm (forward primer, 5'-ATAGAAGAGCACTAGGTATTCCGGCCTAAAT CATA-3', and reverse primer, 5'-CATATGTCCGCGGCCCT AGAACTAGAACTAGTGGA-3'). The $7 x M y c$ fragment was amplified from $p U A S T / 7 x M y c$ using the following primers: forward primer, 5'-CGAGGTCGACGGTATCGA-3'; and reverse primer, 5' - CATATGTCCGCGGCCCTAGAACTAGAACTAGTGGA-3'. The detailed DNA sequences are available on request. Each DsRed-positive transformant was isogenized and confirmed by genomic PCR and direct sequencing (the primer sets used for genotyping are listed in Supplemental Fig. S1).

\section{Generation of the truncated form of Eph}

The truncated version of Eph was generated according to previously described methods (Carvalho et al. 2006). The cDNA fragments of the truncated form of Eph were generated by PCR and subcloned in KpnI- and XbaI-digested pUAST/Flag-HA (for in vitro experiments) and $p U A S T$ attB/Flag-HA (for in vivo experiments) containing a signal peptide followed by three HA tags (the Flag tag was removed by digestion). To perform the MARCM rescue experiment in vivo, the Eph-shRNA-resistant Eph cDNA was used as a template. The detailed DNA sequences are available on request.

\section{Mosaic analysis}

MARCM analysis was performed according to previously described methods (Lee and Luo 1999; Potter et al. 2010). We generated the PN neuroblast clones by exposing flies with the appropriate genotypes to a $1-\mathrm{h}$ heat shock $\left(37^{\circ} \mathrm{C}\right)$ at $0-24 \mathrm{~h}$ after larval hatching, with the exception of 1.5 -h heat shock delivered twice with a 1-h interval for independent double MARCM. We dissected both male and female flies within a week following eclosion, with the exceptions described in Supplemental Figure S4.

\section{Immunostaining}

The immunostaining of fly brains was performed as described previously (Wu and Luo 2006). We used rat anti-mCD8 (1:200;
Invitrogen, MCD0800), mouse anti-Bruchpilot (1:50; Developmental Studies Hybridoma Bank [DSHB], nc82), mouse antimyc (1:1000; Invitrogen, 46-0603), rat anti- $D N$-cadherin (1:50; DSHB, DNEX-8), rabbit anti-GFP (1:500; MBL, 598), mouse anti-HA (1:1000; Covance, 16B12), and goat anti-tdTomato (1:200; Sicgen, AB8181-200) antibodies. Drosophila S2 cells were cultured on concanavalin A-coated coverslips $\left(25^{\circ} \mathrm{C}\right)$, transferred to $4^{\circ} \mathrm{C}$, incubated for $20 \mathrm{~min}$ with $2 \%$ bovine serum albumin (BSA) in phosphate-buffered saline (PBS) followed by a 1-h incubation with $1 \mu \mathrm{g} / \mathrm{mL}$ Ephrin-Fc, and fixed. The immunostaining was performed with mouse anti-HA (1:1000; Covance, 16B12) and goat anti-human-IgG (Fc specific)-Cy3 (1:1000, Sigma-Aldrich, C2571) antibodies. The immunofluorescence signals were acquired using a TCS SP5 or SP8 confocal scanning microscope (Leica) and then processed using ImageJ and Adobe Photoshop.

\section{Coimmunoprecipitation and immunoblotting}

The actin-Gal4 and desired UAS constructs were cotransfected using Effectene transfection reagent (Qiagen). Drosophila S2 cells were plated at a density of $6.0 \times 10^{6}$ cells in a $60-\mathrm{mm}$ dish and transfected according to the manufacturer's protocol. The cells were collected $48 \mathrm{~h}$ after transfection, sonicated in lysis buffer (25 mM Tris- $\mathrm{HCl}$ at $\mathrm{pH} 7.9,10 \mathrm{mM} \mathrm{NaCl}, 2$ mM EDTA, 0.5\% Triton X-100, $10 \mathrm{mM}$ DTT, $1 \times$ Complete [Roche]), and incubated with Ephrin-Fc for $1 \mathrm{~h}$. Next, protein G agarose (Roche) was added and immunoprecipitated according to the manufacturer's protocol. Western blotting was performed with the mouse anti-HA antibody according to standard techniques (1:1000; Covance, 16B12).

\section{Statistical analysis}

For the quantification of VAld dendritic spillover in Supplemental Figure 5, we used the Student's $t$-test. The sample sizes and $P$-value are indicated in either the figure or figure legend. The sample sizes were chosen according to standard practice in the field.

\section{Acknowledgments}

We thank N. Perrimon (Harvard Medical School) for U6B$s g R N A$-short plasmid, and S. Kondo (National Institute of Genetics) for $p H D$-DsRed-attP plasmid. We thank J. Thomas (Salk Institute) for $p C E P 4 / E p h r i n-F c$ plasmid and $E p h^{X 652}$ stock, and the Kyoto Stock Center (DGGR) and Bloomington Drosophila Stock Center for fly stocks. We thank all of the members of the Miura laboratory for their comments on this study. We especially thank S. Haraguchi, C. Sakuma, M. Okumura, K. Takeuchi, T. Umehara, H. Moriya, and Y. Fujisawa for help dissecting $>6000$ flies for the independent double-MARCM experiment. This study was supported by grants from the Ministry of Education, Culture, Sports, Science, and Technology in Japan to M.M. and T.C.; the Japan Society for the Promotion of Science to M. A., S.S., M.M., and T.C.; the Japan Science and Technology Agency to M.M. and T.C.; the Japan Agency for Medical Research and Development (AMED)-CREST; AMED to M.M.; and the Naito Foundation to T.C.

\section{References}

Bossing T, Brand AH. 2002. Dephrin, a transmembrane ephrin with a unique structure, prevents interneuronal axons from 
exiting the Drosophila embryonic CNS. Development 129: 4205-4218.

Boyle M, Nighorn A, Thomas JB. 2006. Drosophila Eph receptor guides specific axon branches of mushroom body neurons. Development 133: 1845-1854.

Cang J, Feldheim DA. 2013. Developmental mechanisms of topographic map formation and alignment. Annu Rev Neurosci 36: 51-77.

Carvalho RF, Beutler M, Marler KJ, Knoll B, Becker-Barroso E, Heintzmann R, Ng T, Drescher U. 2006. Silencing of EphA3 through a cis interaction with ephrinA5. Nat Neurosci 9: 322-330.

Chin-Sang ID, Moseley SL, Ding M, Harrington RJ, George SE, Chisholm AD. 2002. The divergent C. elegans ephrin EFN-4 functions in embryonic morphogenesis in a pathway independent of the VAB-1 Eph receptor. Development 129: 54995510.

Chou YH, Spletter ML, Yaksi E, Leong JC, Wilson RI, Luo L. 2010. Diversity and wiring variability of olfactory local interneurons in the Drosophila antennal lobe. Nat Neurosci 13: 439-449.

Couto A, Alenius M, Dickson BJ. 2005. Molecular, anatomical, and functional organization of the Drosophila olfactory system. Curr Biol 15: 1535-1547.

Datta SR, Vasconcelos ML, Ruta V, Luo S, Wong A, Demir E, Flores J, Balonze K, Dickson BJ, Axel R. 2008. The Drosophila pheromone cVA activates a sexually dimorphic neural circuit. Nature 452: 473-477.

Dearborn R Jr, He Q, Kunes S, Dai Y. 2002. Eph receptor tyrosine kinase-mediated formation of a topographic map in the Drosophila visual system. J Neurosci 22: 1338-1349.

Ejima A, Smith BP, Lucas C, van der Goes van Naters W, Miller CI, Carlson JR, Levine JD, Griffith LC. 2007. Generalization of courtship learning in Drosophila is mediated by cis-vaccenyl acetate. Curr Biol 17: 599-605.

Fishilevich E, Vosshall LB. 2005. Genetic and functional subdivision of the Drosophila antennal lobe. Curr Biol 15: 1548-1553.

Flanagan JG. 2006. Neural map specification by gradients. Curr Opin Neurobiol 16: 59-66.

Gratz SJ, Ukken FP, Rubinstein CD, Thiede G, Donohue LK, Cummings AM, O'Connor-Giles KM. 2014. Highly specific and efficient CRISPR/Cas9-catalyzed homology-directed repair in Drosophila. Genetics 196: 961-971.

Grosjean Y, Rytz R, Farine JP, Abuin L, Cortot J, Jefferis GS, Benton R. 2011. An olfactory receptor for food-derived odours promotes male courtship in Drosophila. Nature 478: 236240.

Hong W, Luo L. 2014. Genetic control of wiring specificity in the fly olfactory system. Genetics 196: 17-29.

Hong W, Zhu H, Potter CJ, Barsh G, Kurusu M, Zinn K, Luo L. 2009. Leucine-rich repeat transmembrane proteins instruct discrete dendrite targeting in an olfactory map. Nat Neurosci 12: $1542-1550$.

Jefferis GS, Hummel T. 2006. Wiring specificity in the olfactory system. Semin Cell Dev Biol 17: 50-65.

Jefferis GS, Vyas RM, Berdnik D, Ramaekers A, Stocker RF, Tanaka NK, Ito K, Luo L. 2004. Developmental origin of wiring specificity in the olfactory system of Drosophila. Development 131: 117-130.

Kandler K, Clause A, Noh J. 2009. Tonotopic reorganization of developing auditory brainstem circuits. Nat Neurosci 12: 711-717.
Kaneko M, Nighorn A. 2003. Interaxonal Eph-ephrin signaling may mediate sorting of olfactory sensory axons in Manduca sexta. J Neurosci 23: 11523-11538.

Kania A, Klein R. 2016. Mechanisms of ephrin-Eph signalling in development, physiology and disease. Nat Rev Mol Cell Biol 17: 240-256.

Kao TJ, Kania A. 2011. Ephrin-mediated cis-attenuation of Eph receptor signaling is essential for spinal motor axon guidance. Neuron 71: 76-91.

Klein R. 2012. Eph/ephrin signalling during development. Development 139: 4105-4109.

Knoll B, Zarbalis K, Wurst W, Drescher U. 2001. A role for the EphA family in the topographic targeting of vomeronasal axons. Development 128: 895-906.

Komiyama T, Sweeney LB, Schuldiner O, Garcia KC, Luo L. 2007. Graded expression of semaphorin-1a cell-autonomously directs dendritic targeting of olfactory projection neurons. Cell 128: 399-410.

Kurtovic A, Widmer A, Dickson BJ. 2007. A single class of olfactory neurons mediates behavioural responses to a Drosophila sex pheromone. Nature 446: 542-546.

Lebreton S, Grabe V, Omondi AB, Ignell R, Becher PG, Hansson BS, Sachse S, Witzgall P. 2014. Love makes smell blind: mating suppresses pheromone attraction in Drosophila females via Or65a olfactory neurons. Sci Rep 4: 7119.

Lee T, Luo L. 1999. Mosaic analysis with a repressible cell marker for studies of gene function in neuronal morphogenesis. Neuron 22: 451-461.

Lin HH, Cao DS, Sethi S, Zeng Z, Chin JS, Chakraborty TS, Shepherd AK, Nguyen CA, Yew JY, Su CY, et al. 2016. Hormonal modulation of pheromone detection enhances male courtship success. Neuron 90: 1272-1285.

Lisle JE, Mertens-Walker I, Rutkowski R, Herington AC, Stephenson SA. 2013. Eph receptors and their ligands: promising molecular biomarkers and therapeutic targets in prostate cancer. Biochim Biophys Acta 1835: 243-257.

Liu W, Liang X, Gong J, Yang Z, Zhang YH, Zhang JX, Rao Y. 2011. Social regulation of aggression by pheromonal activation of Or65a olfactory neurons in Drosophila. Nat Neurosci 14: 896-902.

Potter CI, Tasic B, Russler EV, Liang L, Luo L. 2010. The Q system: a repressible binary system for transgene expression, lineage tracing, and mosaic analysis. Cell 141: 536-548.

Ren X, Sun J, Housden BE, Hu Y, Roesel C, Lin S, Liu LP, Yang Z, Mao D, Sun L, et al. 2013. Optimized gene editing technology for Drosophila melanogaster using germ line-specific Cas9. Proc Natl Acad Sci 110: 19012-19017.

Sakano H. 2010. Neural map formation in the mouse olfactory system. Neuron 67: 530-542.

Sakuma C, Anzo M, Miura M, Chihara T. 2014. Development of olfactory projection neuron dendrites that contribute to wiring specificity of the Drosophila olfactory circuit. Genes Genet Syst 89: 17-26.

Sakurai T, Namiki S, Kanzaki R. 2014. Molecular and neural mechanisms of sex pheromone reception and processing in the silkmoth Bombyx mori. Front Physiol 5: 125.

Scully AL, McKeown M, Thomas JB. 1999. Isolation and characterization of Dek, a Drosophila eph receptor protein tyrosine kinase. Mol Cell Neurosci 13: 337-347.

Seiradake E, Harlos K, Sutton G, Aricescu AR, Jones EY. 2010. An extracellular steric seeding mechanism for Eph-ephrin signaling platform assembly. Nat Struct Mol Biol 17: 398-402.

Sekine SU, Haraguchi S, Chao K, Kato T, Luo L, Miura M, Chihara T. 2013. Meigo governs dendrite targeting specificity by 
modulating ephrin level and N-glycosylation. Nat Neurosci 16: 683-691.

Silbering AF, Rytz R, Grosjean Y, Abuin L, Ramdya P, Jefferis GS, Benton R. 2011. Complementary function and integrated wiring of the evolutionarily distinct Drosophila olfactory subsystems. J Neurosci 31: 13357-13375.

Singh AP, Das RN, Rao G, Aggarwal A, Diegelmann S, Evers JF, Karandikar H, Landgraf $M$, Rodrigues V, Vijayraghavan K. 2013. Sensory neuron-derived eph regulates glomerular arbors and modulatory function of a central serotonergic neuron. PLoS Genet 9: e1003452.

van der Goes van Naters W, Carlson JR. 2007. Receptors and neurons for fly odors in Drosophila. Curr Biol 17: 606612.
Ward A, Hong W, Favaloro V, Luo L. 2015. Toll receptors instruct axon and dendrite targeting and participate in synaptic partner matching in a Drosophila olfactory circuit. Neuron 85: 1013-1028.

Wu JS, Luo L. 2006. A protocol for dissecting Drosophila melanogaster brains for live imaging or immunostaining. Nat Protoc 1: 2110-2115.

Zhu H, Luo L. 2004. Diverse functions of N-cadherin in dendritic and axonal terminal arborization of olfactory projection neurons. Neuron 42: 63-75.

Zhu H, Hummel T, Clemens JC, Berdnik D, Zipursky SL, Luo L. 2006. Dendritic patterning by Dscam and synaptic partner matching in the Drosophila antennal lobe. Nat Neurosci 9: 349-355. 


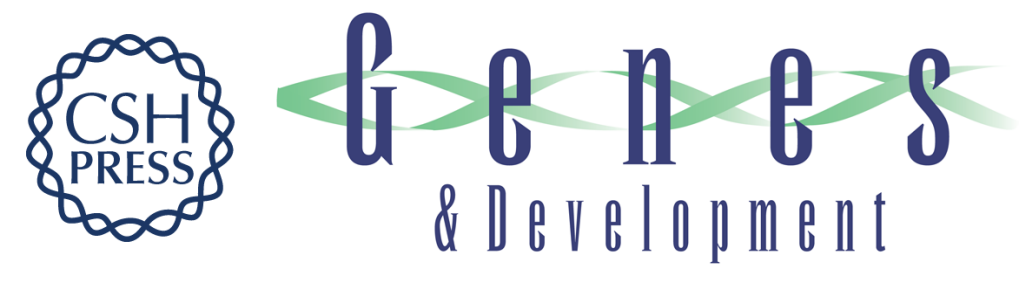

\section{Dendritic Eph organizes dendrodendritic segregation in discrete olfactory map formation in Drosophila}

Marie Anzo, Sayaka Sekine, Shirin Makihara, et al.

Genes Dev. 2017, 31:

Access the most recent version at doi:10.1101/gad.297424.117

Supplemental
Material http://genesdev.cshlp.org/content/suppl/2017/06/21/31.10.1054.DC1

References This article cites 46 articles, 11 of which can be accessed free at:

http://genesdev.cshlp.org/content/31/10/1054.full.html\#ref-list-1

Creative This article is distributed exclusively by Cold Spring Harbor Laboratory Press for the first Commons

License

Email Alerting

Service six months after the full-issue publication date (see

http://genesdev.cshlp.org/site/misc/terms.xhtml). After six months, it is available under a Creative Commons License (Attribution-NonCommercial 4.0 International), as described at http://creativecommons.org/licenses/by-nc/4.0/.

Receive free email alerts when new articles cite this article - sign up in the box at the top right corner of the article or click here.

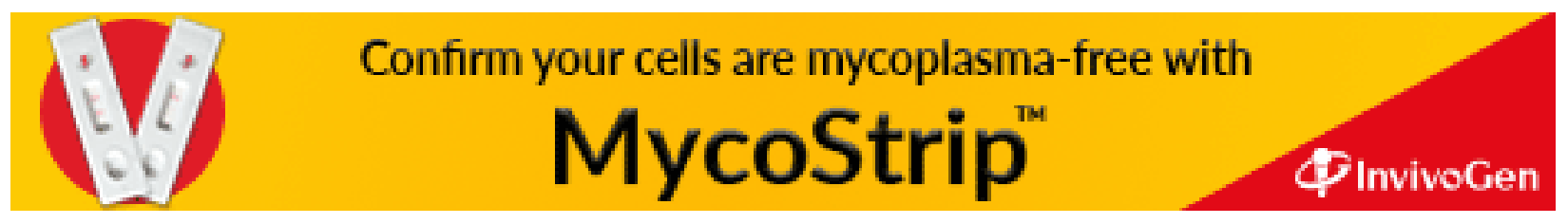

